\title{
Synthesis of Graphene-based Materials for Surface- Enhanced Raman Scattering Applications
}

\author{
Seiya Suzuki ${ }^{\dagger}$ \\ International Center for Young Scientists (ICYS), National Institute for Materials Science (NIMS), 1-1 Namiki, Tsukuba, Ibaraki 305-0044, \\ Japan \\ †Corresponding author: seiya09417@gmail.com
}

Received: 25 April, 2019; Accepted: 29 June, 2019; Published: 25 July, 2019

Raman spectroscopy provides a meaningful fingerprint for sensing and discriminating materials, and surface-enhanced Raman scattering (SERS) can dramatically increase Raman signals up to the single-molecule level of sensitivity. Graphene, a monolayer carbon sheet, has recently attracted considerable attention as a unique SERS substrate. However, there are various types of graphene materials, and the SERS application category is significantly correlated to the structure and quality of the graphene. This review provides a broad perspective on this research area, intended for researchers of diverse fields. First, we categorize the graphene-based SERS applications based on their structure. Second, we introduce the types of graphene (graphene oxide,

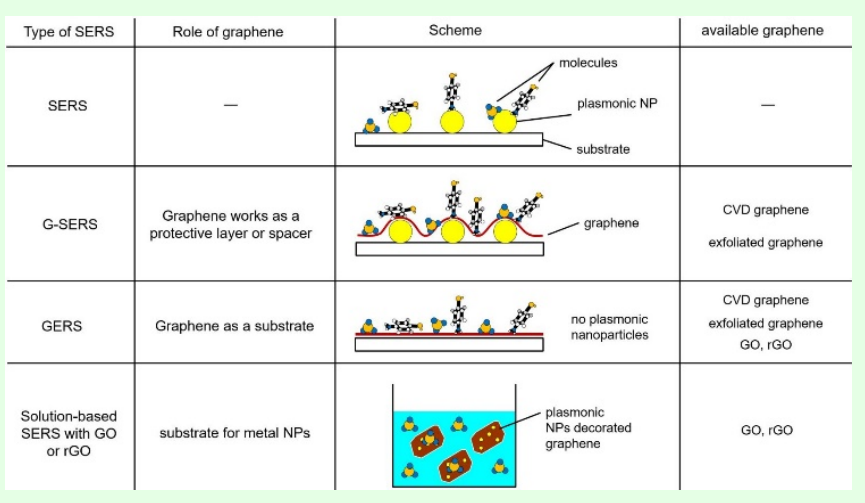
reduced graphene oxide, chemical vapor deposited graphene, and carbon nanowalls) and their synthesis methods. Thereafter, we highlight state-of-the-art studies for each category of graphene-based SERS.

Keywords SERS; Graphene; Graphene oxide; Reduced graphene oxide; Carbon nanowalls; Chemical vapor deposition

\section{INTRODUCTION}

\section{A. Surface-enhanced Raman scattering}

Raman spectroscopy is an optical technique used for characterizing the structure of molecules and lattices based on their unique vibration mode energy levels. Although a Raman spectrum is an important fingerprint for sensing and discriminating materials, the signals are weak owing to the low cross section in Raman scattering [1].

SERS can increase the Raman signals, which significantly improves the sensitivity of Raman spectroscopy. The high sensitivity of SERS owes to the enhancement of local electromagnetic fields via localized surface plasmon resonance [2] in a nanostructured metal surface or metal colloids. The main contribution of SERS enhancement is an electromagnetic mechanism (EM) that provides enhancement of up to $10^{10}$-fold [3-5], and an additional minor contribution is a chemical mechanism (CM) with an enhancement of up to $10^{4}$-fold [6]. From the sum of these enhancements, SERS can sense a single molecule $[7,8]$.

The most common elements used with SERS are coinage metals, such as $\mathrm{Ag}, \mathrm{Au}$, and $\mathrm{Cu}$. Roughened metal surfaces $[9,10]$ and metal nanoparticles (NPs) prepared by chemical $[11,12]$ and thin film deposition methods [13] are commonly used for SERS. The structure of SERS applications can be a solid surface or dispersed solution that contains metal NPs.

Because of the ultrahigh sensitivity and the discriminative ability of SERS, SERS is a promising technique for sensing chemical substances and biomolecules of extremely low abundance [14]. The practical applications of SERS are food safety, disease diagnoses, chemical reaction monitoring, etc. In food safety applications, the detection of pesticide residue, bacteria and viruses, heavy metal ions, and food additives are issues. The required limit of detection (LOD) for those toxic substances depends on the types of foods and substances. For instance, the maximum residue limits for pesticides, which are determined by the domestic organizations for each country, or the Codex Alimentarius Commission globally, typically range from $10^{-2}$ to $10^{2}$ parts per million 
(ppm), which roughly corresponds to $0.1-300 \mu \mathrm{M}$ [15]. SERS has demonstrated the detection of various molecules with low LOD found in pesticides, such as thiabendazole $(<10 \mu \mathrm{M})$ [16], parathion $\left(10^{4}-10^{5}\right.$ with the SERS enhancement factor) [17], methyl parathion $(<100 \mu \mathrm{M})$ [18], carbaryl on apples (4.51 ppm) [19], phosmet on tomatoes (2.91 ppm) [19], and azinphos-methyl on tomatoes (2.94 ppm) [19].

In disease diagnoses, breath analysis has become a popular topic. Breath analysis helps in the early detection of diabetes mellitus, high blood pressure, and cancer. Gas chromatography-mass spectrometry (GC-MS) and semiconductor sensors are conventional gas sensing techniques. GC-MS has high sensitivity and selectivity for gasses but takes a long time for measurement. Semiconductor sensors can perform quick sensing but possess less selectivity for gasses. SERS offers fast and easy measurement with high sensitivity and selectivity for gasses, which overcomes the drawbacks of conventional sensing technique.

The required LODs for breath analysis range from ppm to sub-ppm for some gases, such as acetone, isoprene, and propanol, and from parts per billion to parts per trillion for more abundant kinds of gasses, such as ketones, aldehydes, and pentane [20]. Thus, the highly sensitive detection of gasses is quite important. Wong et al. demonstrated that SERS can detect acetone vapor, which is an important volatile organic compound for breath analysis, with a detection limit of $9.5 \mathrm{pg}$ ( $\sim 6400 \mathrm{ppm})$ [21]. Qiao et al. used a metal organic framework, the zeolitic imidazolate framework-8 (ZIF-8), as a coating material for aggregated gold NPs to detect gaseous aldehydes, and achieved a 10 ppb LOD [22]. The advantage of this SERS material is that it slows down the flow rate of the target gasses to promote and retain adsorption, resulting in enhancement of SERS and increased reproducibility.

The monitoring of chemical reactions, including catalytic and photocatalytic reactions by spectroscopy, provides important ideas about its kinetics. Choi et al. demonstrated that time-resolved single-molecule SERS for studying the dimerization of 4-nitrobenzenethiols could also be applied to other photocatalytic organic reactions [23]. They observed a discrete change in the Raman intensity of 4,4'-dimercaptoazobenzene (DMAB), which is an intermediate in the reaction, indicating the creation or annihilation of the molecule by quantized reaction. To detect such events, extremely high sensitivity at the scale of a single molecule is required, and SERS with single-molecule sensitivity can reveal the detailed mechanism and kinetics of chemical reactions.

Thus, noble applications of SERS have been demonstrated. However, nanostructured materials in SERS are fragile and difficult to fabricate in completely the same manner to ensure reproducible results. In addition, maintaining the high sensitivity of SERS for a long time is also difficult. These difficulties have slowed the commercialization and wider use of SERS applications.

\section{B. Graphene}

Graphene is a single atomic sheet of carbon atoms arranged in a honeycomb lattice [24]. Numerous attractive fundamental properties of graphene have been reported, such as an extremely high carrier mobility approaching $200,000 \mathrm{~cm}^{2} \mathrm{~V}^{-1} \mathrm{~s}^{-1}$ [24-28], universal $2.3 \%$ optical absorption from the mid-infrared to UV light range [29], high Young's modulus of $\sim 1 \mathrm{TPa}$ [30], and high chemical stability $[31,32]$. These properties can be utilized in diverse areas, including electronics [24-28, 33-35], optics [36-39], gas molecule sensing [40], and biomedical applications [41].

Recently, graphene has attracted immense attention as a unique SERS substrate [42-45]. The advantages of graphene in SERS applications can be roughly divided into two categories: (a) as a spacer for metal NPs and adsorbed molecules to stabilize Raman signals, and (b) as a substrate for adsorbed molecules or metal NPs to enhance Raman signals. The former spatial separation of graphene blocks any direct chemical bonding between the metal NPs and molecules, resulting in the suppression of unstable peaks from the interactions between metal molecules in normal SERS. Because graphene is only one atom thick and optically transparent, the decrease in the enhancement by EM is minimal compared with other materials such as metal oxides. Simultaneously, graphene works as a protective layer for metal NPs, which can provide a long SERS lifetime. Some types of graphene possess a large surface area and can be synthesized at a low cost of production. Thus, these types of graphene are good supporting materials for metal NPs, which increase the intensity of Raman peaks in SERS applications.

These advantages of graphene-based SERS contribute to practical SERS applications. For example, the suppression of unstable peaks is important in the detection of residual pesticides for food safety, breath analysis for disease diagnosis, and monitoring chemical reactions, because many kinds of molecules can exist in practical application. The chemically inert surface of graphene can prevent the undesirable side reactions of these molecules, enabling rigid and reliable analysis. The long lifetime and low production cost of graphene-based SERS contribute to many kinds of SERS applications.

However, the properties of real graphene, in reference to the graphene obtained by current fabrication methods, are varied and depend strongly on the synthesis method. In addition, the type of graphene affects the properties of the SERS applications. Thus, graphene-based materials, and the types of graphene-based SERS should be categorized. In addition, to produce ideal graphene-based SERS, it is important to know how it is synthesized. To provide a broad perspective on this research area, we categorize graphene-based SERS applications based on their structure in Section I.C, and then introduce types of graphene with their synthesis methods and properties in Section II. Thereafter, we introduce recent works for graphene-based SERS in Section III. 


\section{Materials \\ Structure of applications}

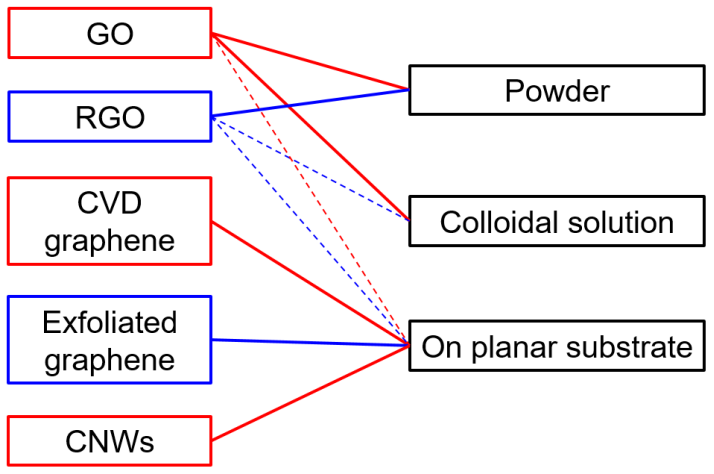

Figure 1: Compatibility between the graphene-based materials and structure of the SERS applications.

\section{Category of SERS applications based on graphene-based materials}

The structures of the SERS applications can be categorized into powder, colloidal solution, and planar substrates. Various types of graphene possess different structures and properties. Therefore, one graphene material may be suitable for a certain SERS application, and another material may not be suitable. To provide compatible combinations of graphene-based materials and SERS applications, a diagram is shown in Figure 1. The listed graphene materials in Figure 1 are graphene oxide (GO), reduced graphene oxide (rGO), Chemical vapor deposition (CVD) graphene, mechanically exfoliated graphene, and carbon nanowalls (CNWs). GO is the oxidized product of bulk graphite, and rGO is its reduced product. CVD graphene is synthesized from carbon gas sources and possesses a higher crystal quality and uniformity than GO or rGO. Exfoliated graphene is obtained by an adhesive tape method from bulk graphite and has a high crystal quality. The disadvantage of exfoliated graphene is the small flake size and uncontrollable layer number. The detailed structures and properties of these graphene materials are explained in Section II. The order of compatibility is represented by solid, broken, and no lines, which show strong, minimal, and weak compatibility, respectively (Figure 1).

GO and rGO can be utilized in powder-based and colloidal solution-based SERS applications. Solution-based SERS can be deposited onto planar substrates by the simple drop-and-dry or spin-coating methods. Powder-based SERS can be dispersed in a solution, and it can also be applied to a planar substrate, as can a solution-based SERS. Therefore, the powder- and solution-based SERS applications with GO and rGO could be commercialized at low cost.

CVD graphene, exfoliated graphene, and CNWs are suitable as planar SERS substrates. Unique and novel properties from CVD and exfoliated graphene-based SERS are expected because of the superior quality and uniformity of graphene compared with that of GO and rGO. The large surface area of the CNWs is beneficial for the decoration of a large number of plasmonic NPs and may contribute to a large enhancement by SERS. However, the cost of fabricating CVD graphene-, exfoliated graphene-, or CNWs-based SERS substrates is expected to be higher than that of GO and $\mathrm{rGO}$, which could be an issue for the commercialization

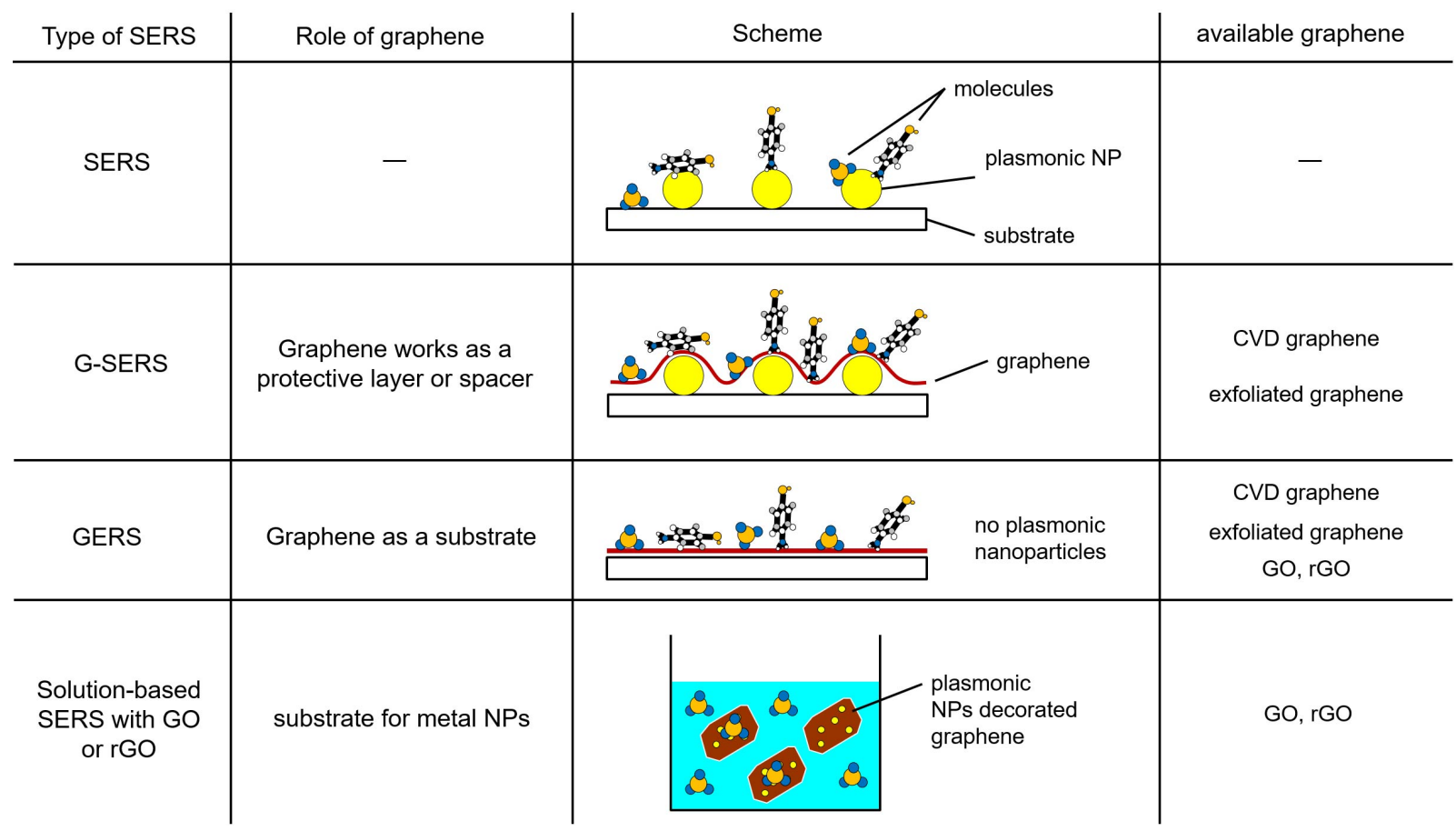

Figure 2: Roles of graphene in G-SERS and GERS and the illustrative structure of SERS, G-SERS, GERS, and solution-based SERS with GO or rGO. 
of their products.

There are two types of planar SERS applications with horizontal graphene: graphene-mediated SERS (G-SERS) and graphene-enhanced Raman scattering (GERS). Figure 2 shows the roles of graphene and the structure of SERS, G-SERS, GERS, and solution-based SERS with GO or rGO. G-SERS was discovered by W. Xu et al. in 2012 [45]. The structural difference between SERS and G-SERS is the graphene inserted between the molecules and the plasmonic NPs. While normal SERS often provides unstable peaks owing to the nonuniform molecular adsorption, charge transfer between the metal and molecules, structural changes of the molecules induced by the laser, or metal-catalyzed side interactions [17], G-SERS does not show this behavior, owing to the spatial separation of the metal-molecule contact. In addition, G-SERS has a high enhancement factor compatible with normal SERS, thus obtaining cleaner and more easily reproducible Raman spectra [32, 45, 46].

The GERS effect was reported first by Ling et al. in 2010 [42]. As shown in Figure 2, GERS does not have plasmonic NPs, indicating that the role of the graphene in GERS is only as a substrate. Because the intrinsic graphene plasmon is in the terahertz range, which is not visible, the enhancement by GERS owes to the CM $[47,48]$. Although the detailed enhancement mechanism of GERS is not fully understood [47], some unique and important features have been reported, such as the effects of the first layer [49], external electrical field [50], and Fermi level [51]. In addition, the easier preparation, lower cost, and better biocompatibility of GERS make it advantageous as a practical SERS substrate [42].

GO and rGO can also work as a GERS substrate. Yu et al. reported that mildly reduced GO showed better enhancement of Raman signals than exfoliated graphene or fully reduced GO [52]. In solution-based SERS applications, GO (or rGO) are often decollated by metal NPs and dispersed in solution, as shown in Figure 2. In this case, the molecules can adsorb on metal NPs and GO (rGO) simultaneously. Although the Raman signals from solution-based GO (rGO) SERS are the result of enhancement by EM and CM from metal NPs and by CM from GO (rGO), the majority of the contribution is from metal NPs.

\section{GRAPHENE-BASED MATERIALS}

\section{A. Mechanically exfoliated graphene}

High-quality graphene can be obtained from bulk graphite by mechanical exfoliation with a commonly used adhesive tape, as reported by A. Geim and K. S. Novoseolov in 2004 [25]. Although exfoliated graphene has been used in primitive GERS [42, 52, 53] and G-SERS [45] studies, the exfoliation method is not suitable for mass production owing to its inherent disadvantages, such as low yield, uncontrollable layer number, and comparatively small flake size [24-28]. Therefore, substitute scalable fabrication of graphene has been studied.

\section{B. GO and rGO}

$\mathrm{GO}$ is a material analogous to graphene. GO is synthesized by oxidizing graphite powders using strong oxidation agents (such as $\mathrm{H}_{2} \mathrm{SO}_{4}, \mathrm{KMnO}_{4}$, and $\mathrm{HNO}_{3}$ ) followed by the liquid exfoliation of individual sheets $[54,55]$. During oxidation, oxygen-containing functional groups (hydroxyl, epoxy, carbonyl, and carboxyl groups), defects, and vacancies are induced in the $\mathrm{sp}^{2}$ carbon network of the graphite [55], as is schematically shown in Figure 3 (at the bottom left). The introduced functional groups reduce the van der Waals forces and increase the interlayer spacing between the adjacent layers, which exfoliates the layers in monolayers by intercalation of the solvent molecules (i.e., $\mathrm{H}_{2} \mathrm{O}$ ) [55-57].

The resultant GO powders are easily dispersible in water; however, they are not electrically conductive. Reduction of the GO is known as an effective method for improving conductivity, and the reduced product is referred to as $\mathrm{rGO}$ or chemically converted graphene (CCG) [58]. Although the conductivity of GO (or rGO) is not directly relevant to the properties of SERS applications, the degree of reduction by GO affects the enhancement of Raman signals, as mentioned in Section I.C. This indicates that the enhancement of GO (or rGO) can be tuned by the reduction process, and the conductivity of rGO as well as its mobility can be used to understand how rGO resembles intrinsic graphene as a crystal.

The methods of reduction, which involve the removal of oxygen functional groups from $\mathrm{GO}$, are roughly divided into chemical (immersed in a solution, such as hydrazine [59] and ascorbic acid [60]) and thermal annealing methods [61]. The thermal annealing of GO in the presence of carbon-containing gasses, referred to as restoration, improves the quality of GO by repairing its defects with the additional carbon atoms $[61,62]$. The conductivity of the obtained rGO is generally lower than that of graphite (graphene) and high-

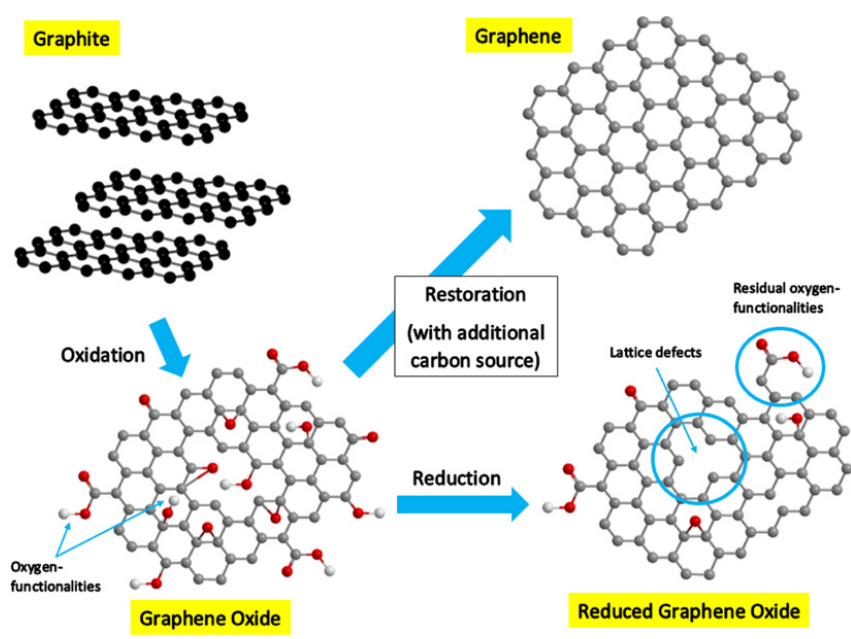

Figure 3: Schematic illustration of the chemical structure of graphite, graphene, graphene oxide, and reduced graphene oxide. [Reprinted with permission from Ref. 61. Copyright 2018, The Japan Society of Applied Physics.] 
er than that of GO. The conductivities of $\mathrm{GO}, \mathrm{rGO}$, and graphite are $10^{-8}-10^{-5} \mathrm{~S} \mathrm{~m}^{-1}[63,64], 5.7 \times 10^{4} \mathrm{~S} \mathrm{~m}^{-1}$ [65], and $\sim 2 \times 10^{6} \mathrm{~S} \mathrm{~m}^{-1}$ [66], respectively. Field-effect transistors (FETs) of rGO have also been fabricated. Although the carrier mobility of rGO is typically $1-10 \mathrm{~cm}^{2} \mathrm{~V}^{-1} \mathrm{~s}^{-1}[67,68]$, recent findings $\left(\sim 900 \mathrm{~cm}^{2} \mathrm{~V}^{-1} \mathrm{~s}^{-1}\right)$ by Obata and Saiki showed the high potential of rGO in electrical applications, as well as in G-SERS applications [69].

The reduction process of GO also affects its dispersibility; $\mathrm{GO}$ is well-dispersed in water, however, rGO becomes an unstable suspension. The dispersibility of rGO is important to its application. For instance, Huang et al. reported that mildly reduced GO can form a uniform membrane film by filtration, while sufficiently reduced GO results in incomplete films or crumbled structures [70]. The main reason for the poor dispersibility of $\mathrm{rGO}$ is the removal of phenolic $\mathrm{OH}$ groups from $\mathrm{GO}$ [71]. Because the removal of phenolic $\mathrm{OH}$ groups is essential to the reduction process in GO, there is a significant trade-off between conductivity and dispersibility in rGO. Thus, the degree of GO reduction should be considered for each application.

\section{CVD graphene}

Another scalable method for producing graphene is CVD. CVD can produce large-area and uniform polycrystalline graphene on catalytic metal surfaces, such as $\mathrm{Cu}$ [72], $\mathrm{Ni}$ [73], Pt [74], Ir [75], and Ru [76]. Among the various catalytic metals, polycrystalline $\mathrm{Cu}$ is the most commonly used substrate for CVD graphene because of its advantages, such as relatively low cost and controllable monolayer growth owing to its low carbon solubility [73]. The CVD growth of graphene on $\mathrm{Cu}$ is modeled as follows: (i) catalytic decomposition of the carbon sources (i.e., methane [72], ethane [77], propane [77], and ethanol vapor [78]) on the $\mathrm{Cu}$ surface); (ii) surface diffusion of the decomposed active species; (iii) nucleation by condensation of the active species; and (iv) domain growth by attachment of the active species to the nucleus $[79,80]$.

CVD generally produces polycrystalline graphene on $\mathrm{Cu}$ $[81,82]$. Because the grain boundaries of graphene degrade its electronic properties, such as carrier mobility [83], reducing the grain boundaries with the growth of large-sized single crystal graphenes (SCGs) is an important area of research. In G-SERS substrates, the undesirable penetration of molecules can occur at the grain boundaries of graphene, which allows the adsorption of molecules on metal NPs, and quashes the role of graphene as a spacer.

The basic strategy for obtaining large-sized SCGs is to reduce the nucleation density. A typical CVD process for obtaining large-sized SCGs on $\mathrm{Cu}$ consists of annealing the $\mathrm{Cu}$ substrate, growing graphene by introducing the carbon source, and then cooling to room temperature (Figure 4). For growing large-sized SCGs, a low supply of the carbon source and a high temperature $\left(>1000^{\circ} \mathrm{C}\right)$ is favorable; however, it is not sufficient for obtaining millimeter-scale SCGs [81]. Recent studies have focused on the annealing

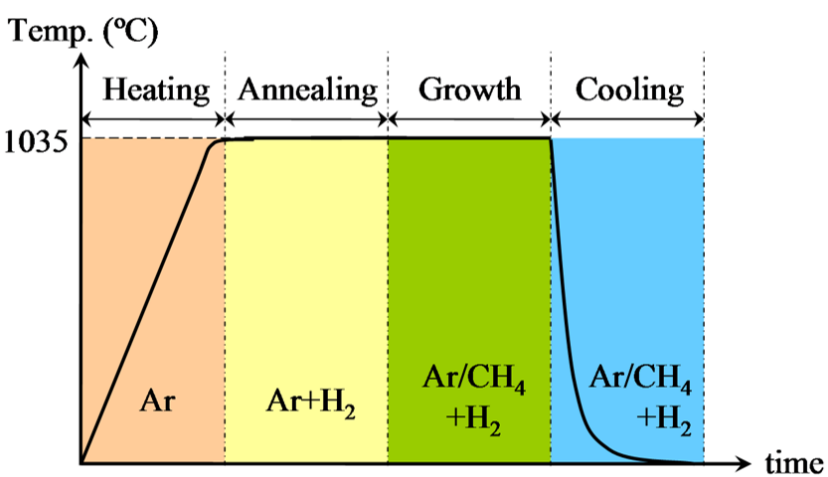

Figure 4: Schematic illustration of a typical CVD growth process. [Reprinted from Ref. 87, licensed under CC BY 4.0.]

step [84, 85], and the improved CVD processes have provided large-sized SCGs at millimeter [85-88] (Figure 5) to centimeter scales $[89,90]$. The carrier mobility of this CVD graphene can reach $65,000 \mathrm{~cm}^{2} \mathrm{~V}^{-1} \mathrm{~s}^{-1}$ and $30,000 \mathrm{~cm}^{2} \mathrm{~V}^{-1}$ $\mathrm{s}^{-1}$ at $1.7 \mathrm{~K}$ and room temperature, respectively, which are comparable to that of exfoliated graphene [89]. The high crystalline quality and the lack of grain boundaries in SCGs completely blocks the undesirable penetration of molecules. Thus, large-sized SCG can be the thinnest and highest-integrity spacer for stabilizing Raman signals in G-SERS.

In addition to its excellent electrical properties, CVD graphene can be transferred onto an arbitrary substrate [91], enabling fabrication for various graphene-based applications [33]. The most common transfer process is polymer-assisted wet transfer [91-93]. The typical wet-transfer process consists of the following steps: (i) spin-coating a supporting polymer onto graphene/Cu; (ii) etching the $\mathrm{Cu}$; (iii) transferring onto a target substrate; and (iv) removing the polymer supporting layer with an organic solvent. Various polymers,
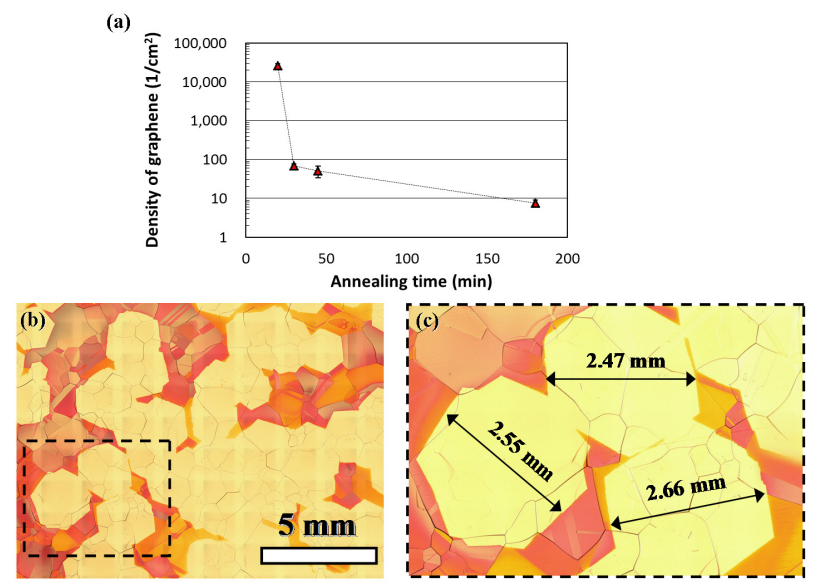

Figure 5: Growth of millimeter-sized SCGs: (a) the dependence of the graphene density on the annealing time, (b) an optical image of the visualized large-size graphene grown at $4 \mathrm{~h}$ of annealing and $3.5 \mathrm{~h}$ of growth, and (c) an enlarged view of (b). [Reprinted from Ref. 87, licensed under CC BY 4.0.] 
such as polymethyl methacrylate (PMMA) [91], polydimethylsiloxane [94], and polycarbonate [95], are available for use as a supporting layer, and PMMA is the most widely used [91-93].

The residues from $\mathrm{Cu}$, its etchant, and the supporting polymer should be avoided on the graphene because they cause degradation in G-SERS and GERS substrates. The attachment of $\mathrm{Cu}$ and its etchant residues to graphene can enlarge the space between the metal NPs and the adsorbed molecules in G-SERS and cause undesirable carrier doping, which can cause fluctuation in the enhancement factor in GERS. The polymer residue provides SERS signals in G-SERS and GERS, presenting difficulty for the analysis of the obtained Raman spectra. Therefore, the transfer process has been improved to provide a cleaner graphene surface.

To etch the $\mathrm{Cu}$ substrate with less residue, $\left(\mathrm{NH}_{4}\right)_{2} \mathrm{~S}_{2} \mathrm{O}_{8}$ should be used, which is a safe and relatively slow $\mathrm{Cu}$ etchant compared with $\mathrm{FeCl}_{3}, \mathrm{FeNO}_{3}, \mathrm{HNO}_{3}$, or $\mathrm{HCl}+\mathrm{H}_{2} \mathrm{O}_{2}[35$, 92]. Wang et al. reported on electrochemical delamination instead of chemical etching of $\mathrm{Cu}$ [96]. In electrochemical delamination, hydrogen bubbles are generated at the graphene $/ \mathrm{Cu}$ interface owing to the reduction of water, and the bubbles push up PMMA/graphene, resulting in the separation of $\mathrm{Cu}$ and graphene. The delamination process is a cost-effective and clean method because the removal of $\mathrm{Cu}$ by delamination is quicker than etching. The $\mathrm{Cu}$ substrate is reusable, and there is no contamination of $\mathrm{Cu}$ or its etchant. To minimize the residue of PMMA, Jeong et al. reported that mild UV irradiation caused moderate degradation of PMMA, and UV irradiated-PMMA could be effectively removed with a mixture of isopropyl alcohol, methyl isobutyl ketone, and acetone [93]. To eliminate the residue of the supporting polymer, a polymer-free transfer of graphene was reported [97, 98]. For instance, Zhang et al. reported on the etching of $\mathrm{Cu}$ at the interface between hexane and an aqueous etchant layer $\left[\left(\mathrm{NH}_{4}\right)_{2} \mathrm{~S}_{2} \mathrm{O}_{8}\right]$ without a solid supporting layer, which stabilizes and protects the freestanding graphene sheet from shear stress [99]. The resultant freestanding graphenes were successfully transferred onto an $\mathrm{SiO}_{2} / \mathrm{Si}$ wafer, a tip for conductive atomic force microscopy (AFM), and metal grids for transmission electron microscopy (TEM) observation. Although numerous challenges such as yield, cleanliness, and automation still need to be addressed, the transfer process for graphene has been improved.

\section{Vertically standing graphene (CNWs)}

Unique graphitic sheets that stand vertically on a flat substrate were discovered by $\mathrm{Wu}$ et al. in 2002 during the growth of carbon nanotubes [100]. Similar types of standing graphitic sheets have been reported by several groups, and they are referred to with various names, such as vertical graphenes [101, 102], carbon nanosheets [103], and CNWs [100]. Here, we refer to them as CNWs based on Wu et al. who first discovered and named them.

The apparent structure and size of the CNWs are schematically shown in Figure 6. Each individual $\mathrm{CNW}$ sheet consists of numerous nanographite domains with an average size of $\sim 20 \mathrm{~nm}$ [104]. The nanographite domains are well aligned, and the plane of the CNW sheet is nearly parallel to the [0001] direction of the graphite [104, 105]. The height of the CNWs typically ranges from $1 \mu \mathrm{m}$ to $2 \mu \mathrm{m}[106,107]$, while their thickness is less than $100 \mathrm{~nm}$ [105] to $2 \mathrm{~nm}$ [108].

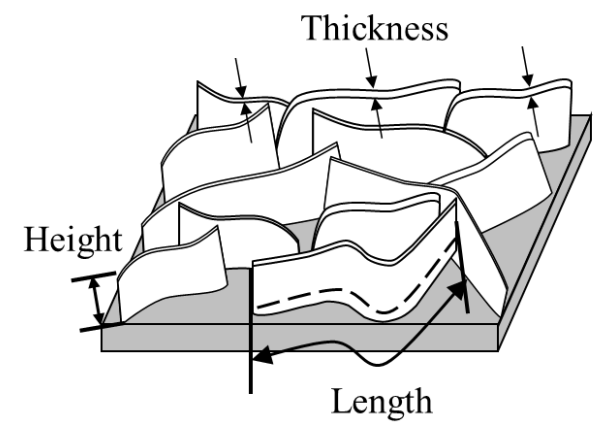

Figure 6: Schematic illustration of the CNWs and definition of their size. [Reprinted with permission from Ref. 106. Copyright 2015, The Japan Society of Applied Physics.]

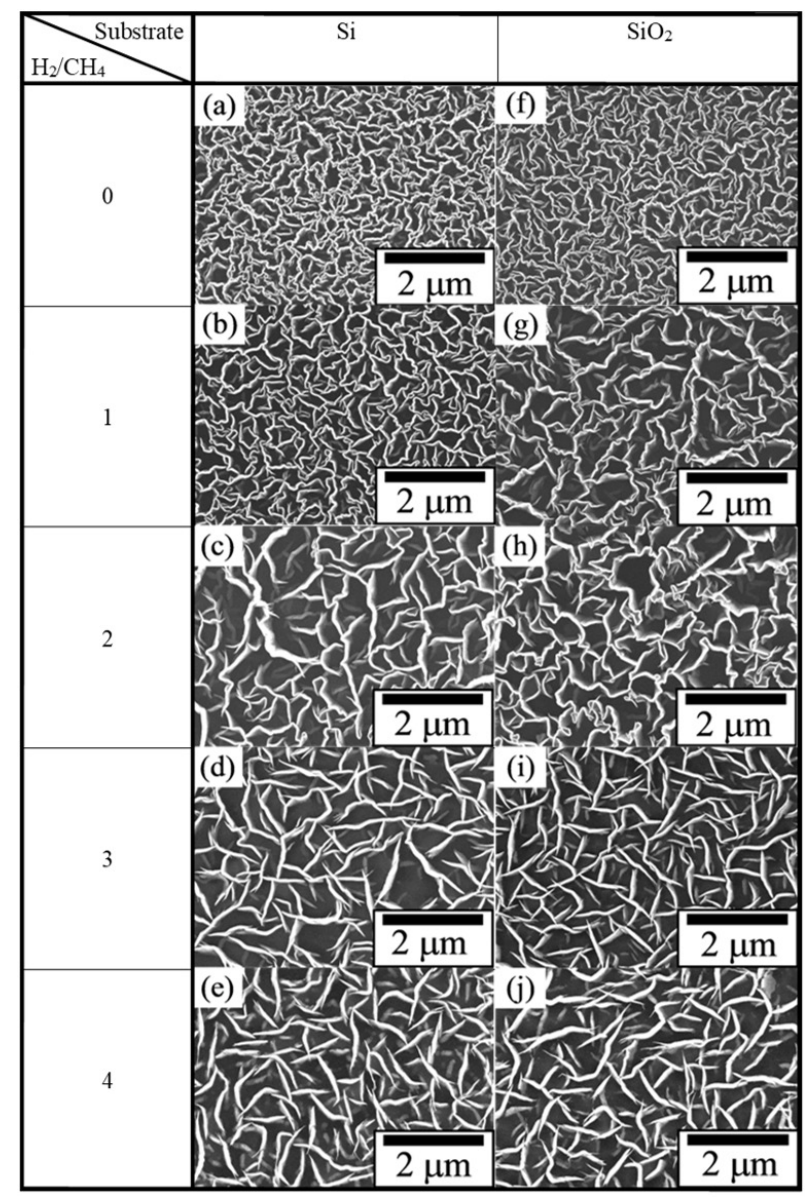

Figure 7: Representative top-view SEM images of the CNWs grown on $\mathrm{Si}$ and $\mathrm{SiO}_{2}$ at different $\mathrm{H}_{2} / \mathrm{CH}_{4}$ flow rate ratios. The shape of the CNWs changed from wavy to straight by increasing the $\mathrm{H}_{2} / \mathrm{CH}_{4}$ ratio. [Reprinted with permission from Ref. 106. Copyright 2015, The Japan Society of Applied Physics.] 

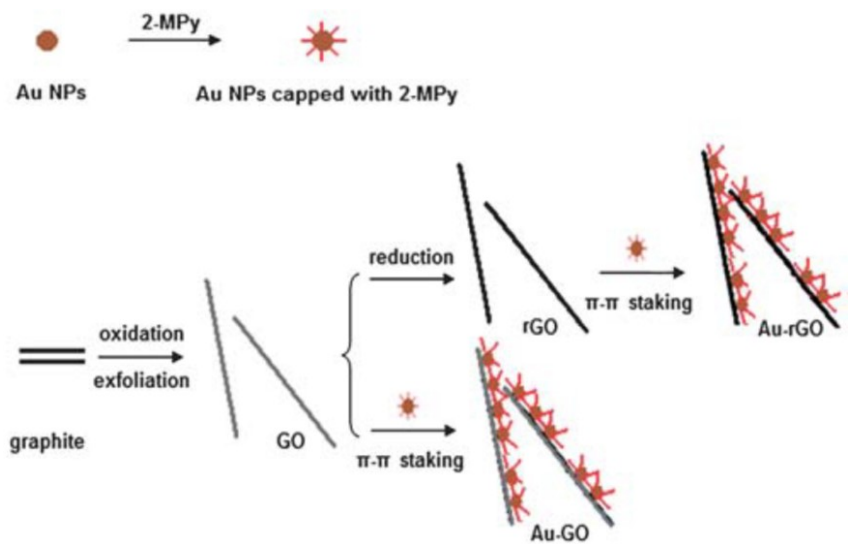

Figure 8: Schematic illustration of the synthesis of Au-GO and Au-rGO composites. [Reprinted with permission from Ref. 127. Copyright 2010, The Royal Society of Chemistry.]

The shape of the individual CNW sheets can vary from wavy to straight depending on the growth conditions [106, 109] (Figure 7).

CNWs are generally grown by plasma-enhanced CVD using a gas mixture of hydrocarbon and hydrogen [100-103, 106], and there have been a few reports of the hot-wire CVD growth of CNWs [110-112]. Various catalytic and non-catalytic substrates can be used for $\mathrm{CNW}$ growth, i.e., $\mathrm{Si}$ [106], $\mathrm{SiO}_{2}$ [105, 106], $\mathrm{Al}_{2} \mathrm{O}_{3}$ [113], $\mathrm{Cu}$ [114], and $\mathrm{Ni}$ [115]. The choice of substrates for CNW growth enables different types of applications, such as for catalyst supports [116], electron field emitters [109, 117], gas sensors [118], superhydrophobic surfaces [119], and negative electrodes for lithium ion batteries [120].

In SERS applications, CNWs are used as a supporting material for metal NPs. Various methods, such as electrodeposition [121, 122], drop casting of colloidal AuNP solution followed by heating [102], magnetron sputtering [101, 123], and E-beam evaporation [124] have been used to decollate CNWs by AuNPs or AgNPs. The advantage of CNWs-based SERS is their large surface area, which increases the observed enhancement by supporting a large number of densely distributed metal NPs.

\section{GRAPHENE-BASED SERS AND ITS SYNTHESIS}

\section{A. GO- and rGO-based SERS}

As previously mentioned, GO [125-127] and rGO [127, 128] can be used for powder-based and colloidal solution-based SERS applications. Figure 8 schematically shows an example fabrication of GO- and rGO-based SERS materials [127]. The decollation of AuNPs on GO (or rGO) has been achieved by stirring a mixture solution of GO (or rGO) and AuNPs. Powder-based GO/AuNP composites can be obtained by centrifugation of the resultant solution [127]. For rGO-based SERS products, the one-pot reduction method can be used [129]. In the one-pot method, $\mathrm{AgNO}_{3}$

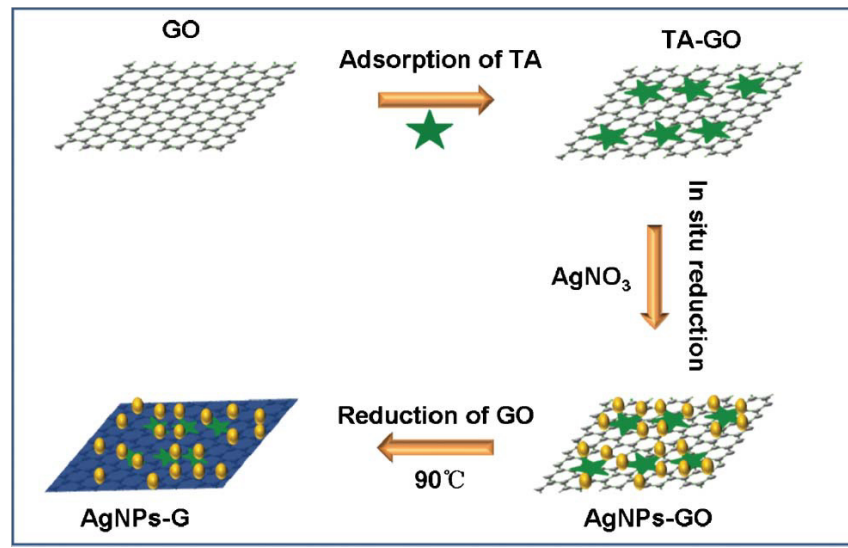

Figure 9: Schematic illustration of the one-pot preparation of AgNP-graphene (AgNP-G) nanocomposites via the chemical reduction of $\mathrm{AgNO}_{3}$ and $\mathrm{GO}$ by tannic acid (TA). [Reprinted with permission from Ref. 130. Copyright 2012, The Royal Society of Chemistry.]

and GO are reduced to AgNPs and rGO in the same container (Figure 9), which is advantageous for production scale and costs [130]. Although the details of the fabrication process differ between studies, chemically synthesized AuNPs [44, 127, 131, 132] and AgNPs [44, 125, 128, 130, 133, 134] are commonly used in GO- and rGO-based SERS. Figure 10 shows the TEM images of $40 \mathrm{~nm}$ AuNPs and $20 \mathrm{~nm}$ AuNPs deposited onto GO sheets and GO, respectively [127]. The typical size of these NPs ranges from several nanometers [129] to several tens of nanometers [127], resulting in enhancement of Raman scattering.

Various types of molecular sensing using GO- and rGO-based SERS have been reported [128, 130, 133]. Zhang et al. reported the sensing of $\mathrm{H}_{2} \mathrm{O}_{2}$ and glucose by
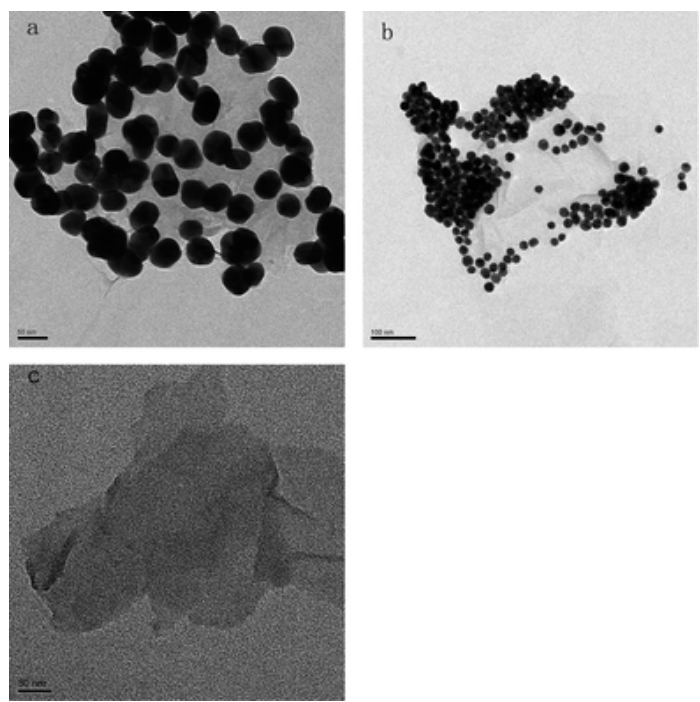

Figure 10: TEM images of (a) $40 \mathrm{~nm}$ AuNPs, (b) $20 \mathrm{~nm}$ AuNPs deposited onto GO sheets, and (c) GO. [Reprinted with permission from Ref. 127. Copyright 2010, The Royal Society of Chemistry.] 
Table 1: Comparison of the enhancement factor and detection limit of R6G with the type of graphene, plasmonic metal, and the wavelength of the incident laser. Exfo-G and CVD-G in the table indicate exfoliated graphene and CVD graphene, respectively.

\begin{tabular}{lccccc}
\hline Type of graphene & Plasmonic metal & Wavelength $(\mathrm{nm})$ & Enhancement factor & Detection limit $(\mathrm{M})$ & Ref. \\
\hline GO & $\mathrm{Ag}$ & 532 & $9.1 \times 10^{9}$ & $1 \times 10^{-12}$ & 125 \\
rGO & $\mathrm{Ag}$ & 785 & $4.9 \times 10^{6}$ & $1 \times 10^{-9}$ & 128 \\
GO & $\mathrm{Ag}$ and Au & 785 & $5.97 \times 10^{8}$ & $1 \times 10^{-13}$ & 126 \\
Exfo-G (GER) & - & 514 & not calculated & $8 \times 10^{-10}$ & 42 \\
CVD-G (GER) & - & 514 & $10^{3}-10^{4}$ & $1 \times 10^{-5}$ & 53 \\
CVD-G (G-SERS) & $\mathrm{Au}$ & 633 & $>10^{10}$ & $1 \times 10^{-14}$ & 137 \\
CVD-G (G-SERS) & $\mathrm{Ag}$ & 532 & not calculated & $<1 \times 10^{-14}$ & 141 \\
CNWs & $\mathrm{Ag}$ & 532 & not calculated & $1 \times 10^{-13}$ & 101 \\
CNWs & $\mathrm{Au}$ & 532 & not calculated & $1 \times 10^{-7}$ & 121 \\
\hline
\end{tabular}

AgNPs/rGO composites with detection limits of $7 \times 10^{-6} \mathrm{M}$ and $1 \times 10^{-4} \mathrm{M}$, respectively [130]. Ren et al. prepared GO/PDDA/AgNPs [PDDA; poly(diallyldimethyl ammonium chloride)] and demonstrated the sensing of folic acid and serum with a detection limit of $9 \times 10^{-9} \mathrm{M}$ in water [133]. PDDA created cationic GO to attract negatively charged molecules and to anchor AgNPs, which provided strong SERS enhancement of folic acid. Jiang et al. increased the SERS enhancement by tuning the loading amount of Ag and sensing a commercial pesticide, paraoxon, by rGOAg-based SERS with a detection limit of $1 \times 10^{-6} \mathrm{M}$ [128]. The rGO-Ag system showed a higher long-term stability [89\% efficiency in the Raman signal of rhodamine $6 \mathrm{G}$ (R6G) after 180 days] than that of bare AgNPs (5.2\% efficiency after 180 days). Xiu et al. reported a three-dimensional flexible SERS substrate using GO/AgNPs on pyramidal-shaped PMMA. The enhancement factor of GO/AgNPs on pyramidal-shaped PMMA for R6G was estimated to reach $8.1 \times 10^{9}$ [125]. Zhang et al. reported a unique ternary hybrid structure of AgNPs, GO, and AuNPs with a high enhancement factor (see Table 1) [126]. Therefore, GO- and rGO-based SERS can sense various molecules, and its sensing ability can be improved by changing the synthesis conditions and structure of the substrate.

\section{B. CVD graphene-based SERS}

Because CVD graphene can cover a large area of the substrate surface with high uniformity in terms of layer numbers and crystallinity, its applications are more practical than those of exfoliated graphene. Although exfoliated graphene has been used in primitive GERS $[42,52,53]$ and G-SERS [45] studies, CVD graphene has been used in recent studies [135-141], probably owing to the improvements in techniques for its growth. Therefore, we focus on CVD graphene-based SERS applications in this section. CVD graphene is expected to demonstrate properties equivalent to exfoliated graphene in SERS applications.

As previously mentioned, CVD graphene can be used for planar SERS applications, such as GER and G-SERS. The preparation of GER substrates involves the transfer of graphene onto a substrate [42], while the preparation of G-SERS substrates also requires metal-NP deposition (Figure 7). To fabricate G-SERS substrates, our group deposited AgNPs on $\mathrm{SiO}_{2} / \mathrm{Si}$ by dc magnetron sputtering, and thereafter, CVD graphene was transferred onto the substrate [135]. Xu et al. first deposited AuNPs or AgNPs on CVD graphene/Cu by vacuum evaporation, and then spin-coated PMMA and removed the backside $\mathrm{Cu}$ layer to fabricate G-SERS substrates, as schematically shown in Figure 11 [45]. These fabrication steps were different; however, both

A

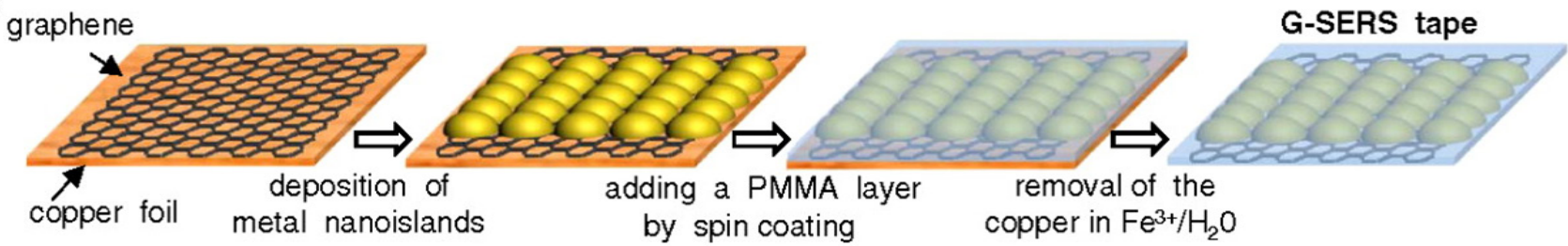

Figure 11: Schematic steps of the preparation of G-SERS tape prepared from CVD monolayer graphene. [Reprinted with permission from Ref. 45. Copyright 2012, National Academy of Sciences.] 
G-SERS substrates had the same configuration, where graphene is located between the plasmonic NPs and molecules.

To produce intense enhancement, the configuration of the G-SERS substrates was further studied. Zhu et al. reported that graphene-covered $\mathrm{Au}$ nanovoid arrays provided a 700-fold enhancement of the Raman signal of graphene [136]. Li et al. reported that a graphene-induced sub-nanometer gap between the AgNPs and Ag film provided a near-field enhancement up to 1700-fold [138]. Wang et al. reported that a graphene-coated pyramidal $\mathrm{Au}$ array provided single-molecule detection of R6G and lysozyme (for enhancement factors greater than $10^{10}$ ) [137].

Flexible substrates for G-SERS have also been developed. $\mathrm{Xu}$ et al. fabricated AgNP-based and AuNP-based G-SERS tapes with transparency and flexibility that could be used in solutions and solids [45]. Leem et al. reported a three-dimensional AuNP-based G-SERS on polystyrene (PS) [139]. The key process involves shrinkage of the PS substrate with the graphene-AuNPs by heating, increasing the enhancement factor to at least 10 times that of the flat substrate. Qiu et al. reported a flexible G-SERS substrate with high enhancement that possessed a detection limit of $<10^{-14} \mathrm{M}$ for R6G [141]. The G-SERS substrate consisted of PMMA/Ag-nanoflowers (AgNFs)/graphene, and the AgNFs were plasmonic metals. The AgNFs were synthesized by microcurrent-assisted chemical reduction of $\mathrm{AgNO}_{3}$, and the well-controlled size and density of the AgNFs resulted in a large enhancement by the G-SERS.

The degradation of the SERS enhancement by long-term storage or repeated use is a crucial issue for its practical application. Our group reported that G-SERS substrates made of a millimeter-sized SCG (Figure 12) showed a high chemical durability for concentrated hydrochloric acid (35-37\%), heated air up to $400^{\circ} \mathrm{C}$, and long-term heating $(>63 \mathrm{~h})$ in air at $150^{\circ} \mathrm{C}$ [135]. Thus, the graphene layer served as a protec- tive layer for the plasmonic NPs. Based on these results, we proposed new types of SERS techniques by G-SERS, such as molecular detection at high temperatures or in extremely acidic conditions.

Thus, a CVD graphene-based planar G-SERS substrate is a practical SERS application with excellent properties, such as a high sensitivity, high mechanical flexibility, and high chemical durability.

\section{CNWs-based SERS}

Although the research for CNW-based SERS is minimal compared to that of other graphene-based SERS, there are some distinguished studies. Wang et al. reported that the detection limit of R6G by CNW-based SERS could reach $10^{-13} \mathrm{M}$ [101], which was a higher sensitivity than that of GO-based and rGO-based SERS in previous studies (see Table 1). Dyakonov et al. reported that AuNP-decollated CNWs could sense biomolecules, such as bovine serum albumin, tryptophan, and guanine, with minimum detection levels of $10^{-6} \mathrm{M}$ [123]. Zhao et al. reported plasmon-driven catalytic reactions on an AgNP-decollated CNW substrate. The reactions were the oxidation of $\mathrm{p}$-aminothiopheno to DMAB and the reduction of 4-nitrobenzenethiol to DMAB [124]. The reaction processes could be traced by SERS. The high surface-to-volume ratio of the CNWs was advantageous for SERS intensity and the yield of the plasmonic chemical reactions relative to the planar graphene-based substrate.

\section{CONCLUSION AND OUTLOOK}

In this review, we introduced and categorized graphene-based materials and SERS applications with their preparation methods. The features of each graphene-based
A

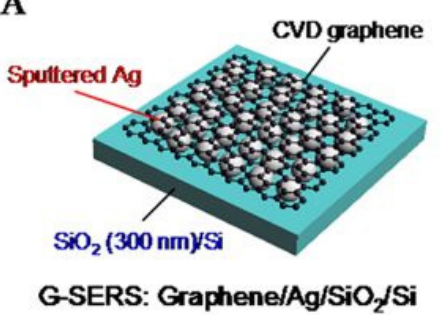

D

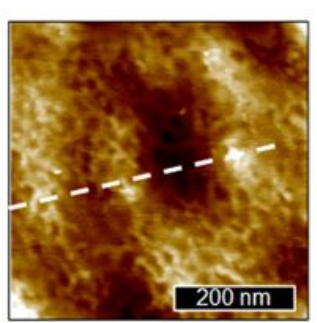

B

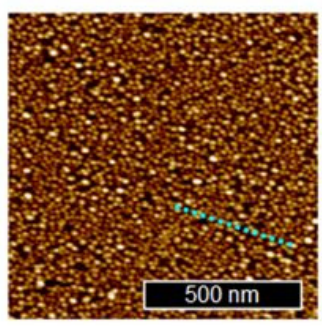

C

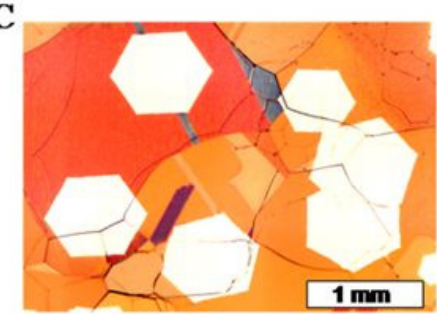

E

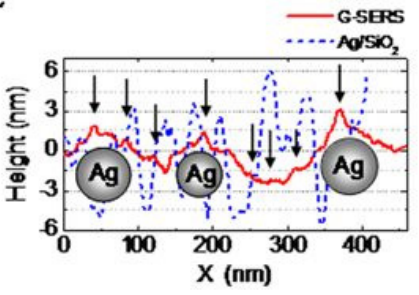

Figure 12: (A) Schematic illustration of the G-SERS substrate. Image (B) shows the AFM image of the AgNPs deposited by sputtering on $\mathrm{SiO}_{2} / \mathrm{Si}$. Image (C) shows the millimeter-sized CVD graphenes on Cu. Image (D) shows the AFM image of the G-SERS substrate. Image (E) shows the AFM line profiles along the broken lines in (B) and (E). [Reprinted with permission from Ref. 135. Copyright 2017, S. Suzuki and M. Yoshimura.] 
SERS are summarized as follows: (i) GO- and rGO-based SERS have advantages for large-scale production at a low cost, and adaptability for powder- or colloidal-based products; (ii) CVD graphene-based SERS possesses superior properties, such as high sensitivity up to the single-molecule detection level, the ability to provide cleaner and more easily reproducible Raman spectra, and high chemical durability; (iii) CNW-based SERS is beneficial for various substrates and large active surface areas, which is an advantage for the yield of plasmonic chemical reactions. Normal SERS substrates have not been widely commercialized owing to the lack of stability, price-performance ratio, and quality. G-SERS substrates have high potential for making SERS a common commercialized product, especially with CVDbased substrates. Further improvement in the properties of graphene-based SERS and the synthesis of graphene-based materials are required. We believe that the concept of graphene-based SERS substrates will be accepted by more researchers in a broad range of fields, such as carbon nanomaterials, biology, sensors, and optics, leading to further development.

\section{References}

[1] P. L. Stiles, J. A. Dieringer, N. C. Shah, and R. P. Van Duyne, Annu. Rev. Anal. Chem. 1, 601 (2008).

[2] M. Moskovits, J. Chem. Phys. 69, 4159 (1978).

[3] P. Johansson, H. Xu, and M. Käll, Phys. Rev. B 72, 035427 (2005).

[4] M. Inoue and K. Ohtaka, J. Phys. Soc. Jpn. 52, 3853 (1983).

[5] Y. S. Yamamoto and T. Itoh, J. Raman Spectrosc. 47, 78 (2016). [6] L. Jensen, C. M. Aikens, and G. C. Schatz, Chem. Soc. Rev. 37, 1061 (2008).

[7] K. Kneipp, Y. Wang, H. Kneipp, L. T. Perelman, I. Itzkan, R. R. Dasari, and M. S. Feld, Phys. Rev. Lett. 78, 1667 (1997).

[8] S. Nie and S. R. Emory, Science 275, 1102 (1997).

[9] J. C. Tsang, J. E. Demuth, P. N. Sanda, and J. R. Kirtley, Chem. Phys. Lett. 76, 54 (1980).

[10] M. Fleischmann, P. J. Hendra, and A. J. McQuillan, Chem. Phys. Lett. 26, 163 (1974).

[11] J. A. Creighton, C. G. Blatchford, and M. G. Albrecht, J. Chem. Soc., Faraday Trans. 2, 75, 790 (1979).

[12] H. Wetzel and H. Gerischer, Chem. Phys. Lett. 76, 460 (1980). [13] D. Büchel, C. Mihalcea, T. Fukaya, N. Atoda, J. Tominaga, T. Kikukawa, and H. Fuji, Appl. Phys. Lett. 79, 620 (2001).

[14] T. Vo-Dinh, Sens. Actuators B Chem. 29, 183 (1995).

[15] Maximum Residue Limits (MRLs) List of Agricultural Chemicals in Foods (Search engine for MRLs, The Japan Food Chemical Research Foundation, http://db.ffcr.or.jp/front/, retrieved on Mar. 31, 2019).

[16] B. Fortuni, Y. Fujita, M. Ricci, T. Inose, R. Aubert, G. Lu, J. A. Hutchison, J. Hofkens, L. Latterini, and H. Uji-i, Chem. Commun. 53, 5121 (2017).

[17] J. F. Li, Y. F. Huang, Y. Ding, Z. L. Yang, S. B. Li, X. S. Zhou, F. R. Fan, W. Zhang, Z. Y. Zhou, D. Y. Wu, B. Ren, Z. L. Wang, and Z. Q. Tian, Nature 464, 392 (2010).

[18] X. Li, G. Chen, L. Yang, Z. Jin, and J. Liu, Adv. Funct. Mater. 20, 2815 (2010).

[19] B. Liu, P. Zhou, X. Liu, X. Sun, H. Li, and M. Lin, Food Bioproc. Tech. 6, 710 (2013).
[20] J. Pereira, P. Porto-Figueira, C. Cavaco, K. Taunk, S. Rapole, R. Dhakne, H. Nagarajaram, and J. S. Câmara, Metabolites 5, 3 (2015).

[21] C. L. Wong, U. Dinish, K. D. Buddharaju, M. S. Schmidt, and M. Olivo, Appl. Phys. A 117, 687 (2014).

[22] X. Qiao, B. Su, C. Liu, Q. Song, D. Luo, G. Mo, and T. Wang, Adv. Mater. 30, 1702275 (2018).

[23] H.-K. Choi, W.-H. Park, C.-G. Park, H.-H. Shin, K. S. Lee, and Z. H. Kim, J. Am. Chem. Soc. 138, 4673 (2016).

[24] A. K. Geim and K. S. Novoselov, Nat. Mater. 6, 183 (2007).

[25] K. S. Novoselov, A. K. Geim, S. V. Morozov, D. Jiang, Y. Zhang, S. V. Dubonos, I. V. Grigorieva, and A. A. Firsov, Science 306, 666 (2004).

[26] K. S. Novoselov, A. K. Geim, S. V. Morozov, D. Jiang, M. I. Katsnelson, I. V. Grigorieva, S. V. Dubonos, and A. A. Firsov, Nature 438, 197 (2005).

[27] Y. Zhang, Y.-W. Tan, H. L. Stormer, and P. Kim, Nature 438, 201 (2005).

[28] P. Avouris, Z. Chen, and V. Perebeinos, Nat. Nanotechnol. 2, 605 (2007).

[29] R. R. Nair, P. Blake, A. N. Grigorenko, K. S. Novoselov, T. J. Booth, T. Stauber, N. M. Peres, and A. K. Geim, Science 320, 1308 (2008).

[30] C. Lee, X. Wei, J. W. Kysar, and J. Hone, Science 321, 385 (2008).

[31] H. Y. Nan, Z. H. Ni, J. Wang, Z. Zafar, Z. X. Shi, and Y. Y. Wang, J. Raman Spectrosc. 44, 1018 (2013).

[32] S. Suzuki and M. Yoshimura, Sci. Rep. 7, 14851 (2017).

[33] X. Li, Y. Zhu, W. Cai, M. Borysiak, B. Han, D. Chen, R. D. Piner, L. Colombo, and R. S. Ruoff, Nano Lett. 9, 4359 (2009).

[34] X. Wang, L. Zhi, and K. Müllen, Nano Lett. 8, 323 (2008).

[35] S. Bae, H. Kim, Y. Lee, X. Xu, J.-S. Park, Y. Zheng, J. Balakrishnan, T. Lei, H. R. Kim, Y. I. Song, Y.-J. Kim, K. S. Kim, B. Özyilmaz, J.-H. Ahn, B. H. Hong, and S. Iijima, Nat. Nanotechnol. 5, 574 (2010).

[36] F. Xia, T. Mueller, Y.-M. Lin, A. Valdes-Garcia, and P. Avouris, Nat. Nanotechnol. 4, 839 (2009).

[37] T. Mueller, F. Xia, and P. Avouris, Nat. Photonics 4, 297 (2010).

[38] M. Liu, X. Yin, E. Ulin-Avila, B. Geng, T. Zentgraf, L. Ju, F. Wang, and X. Zhang, Nature 474, 64 (2011).

[39] C.-C. Lee, S. Suzuki, W. Xie and T. Schibli, Opt. Express 20, 5264 (2012).

[40] N. Harada, K. Hayashi, M. Kataoka, J. Yamaguchi, M. Ohtomo, M. Ohfuchi, I. Soga, D. Kondo, T. Iwai, and S. Sato, 2016 IEEE International Electron Devices Meeting (San Francisco, 2016) 18.2.1.

[41] G. Reina, J. M. González-Domínguez, A. Criado, E. Vázquez, A. Bianco, and M. Prato, Chem. Soc. Rev. 46, 4400 (2017).

[42] X. Ling, L. Xie, Y. Fang, H. Xu, H. Zhang, J. Kong, M. S. Dresselhaus, J. Zhang, and Z. Liu, Nano Lett. 10, 553 (2010).

[43] C. Xu and X. Wang, Small 5, 2212 (2009).

[44] G. Lu, H. Li, C. Liusman, Z. Yin, S. Wu, and H. Zhang, Chem. Sci. 2, 1817 (2011).

[45] W. Xu, X. Ling, J. Xiao, M. S. Dresselhaus, J. Kong, H. Xu, Z. Liu, and J. Zhang, Proc. Natl. Acad. Sci. U.S.A. 109, 9281 (2012).

[46] W. Xu, J. Xiao, Y. Chen, Y. Chen, X. Ling, and J. Zhang, Adv. Mater. 25, 928 (2013).

[47] H. Lai, F. Xu, Y. Zhang, and L. Wang, J. Mater. Chem. B 6, 4008 (2018).

[48] W. Xu, N. Mao, and J. Zhang, Small 9, 1206 (2013).

[49] X. Ling and J. Zhang, Small 6, 2020 (2010). 
[50] H. Xu, Y. Chen, W. Xu, H. Zhang, J. Kong, M. S. Dresselhaus, and J. Zhang, Small 7, 2945 (2011).

[51] H. Xu, L. Xie, H. Zhang, and J. Zhang, ACS Nano 5, 5338 (2011).

[52] X. Yu, H. Cai, W. Zhang, X. Li, N. Pan, Y. Luo, X. Wang, and J. G. Hou, ACS Nano 5, 952 (2011).

[53] S. Huh, J. Park, Y. S. Kim, K. S. Kim, B. H. Hong, and J.-M. Nam, ACS Nano 5, 9799 (2011).

[54] H. He, J. Klinowski, M. Forster, and A. Lerf, Chem. Phys. Lett. 287, 53 (1998).

[55] S. Mao, H. Pu, and J. Chen, RSC Adv. 2, 2643 (2012).

[56] D. Li, M. B. Müller, S. Gilje, R. B. Kaner, and G. G. Wallace, Nat. Nanotechnol. 3, 101 (2008).

[57] D. Yang, A. Velamakanni, G. Bozoklu, S. Park, M. Stoller, R. D. Piner, S. Stankovich, I. Jung, D. A. Field, C. A. Ventrice Jr., and R. S. Ruoff, Carbon 47, 145 (2009).

[58] K. K. H. De Silva, H.-H. Huang, R. K. Joshi, and M. Yoshimura, Carbon 119, 190 (2017).

[59] S. Stankovich, D. A. Dikin, R. D. Piner, K. A. Kohlhaas, A. Kleinhammes, Y. Jia, Y. Wu, S. T. Nguyen, and R. S. Ruoff, Carbon 45, 1558 (2007).

[60] K. K. H. De Silva, H.-H. Huang, and M. Yoshimura, Appl. Surf. Sci. 447, 338 (2018).

[61] K. K. H. De Silva, H.-H. Huang, S. Suzuki, R. Badam, and M. Yoshimura, Jpn. J. Appl. Phys. 57, 08NB03 (2018).

[62] C.-Y. Su, Y. Xu, W. Zhang, J. Zhao, A. Liu, X. Tang, C.-H. Tsai, Y. Huang, and L.-J. Li, ACS Nano 4, 5285 (2010).

[63] H. C. Schniepp, J.-L. Li, M. J. McAllister, H. Sai, M. Herrera-Alonso, D. H. Adamson, R. K. Prud'homme, R. Car, D. A. Saville, and I. A. Aksay, J. Phys. Chem. B 110, 8535 (2006).

[64] H.-J. Shin, K. K. Kim, A. Benayad, S.-M. Yoon, H. K. Park, I.-S. Jung, M. H. Jin, H.-K. Jeong, J. M. Kim, J.-Y. Choi, and Y. H. Lee, Adv. Funct. Mater. 19, 1987 (2009).

[65] S. Dubin, S. Gilje, K. Wang, V. C. Tung, K. Cha, A. S. Hall, J. Farrar, R. Varshneya, Y. Yang, and R. B. Kaner, ACS Nano 4, 3845 (2010).

[66] Y. Kaburagi and Y. Hishiyama, Carbon 33, 773 (1995).

[67] G. Eda, G. Fanchini, and M. Chhowalla, Nat. Nanotechnol. 3, 270 (2008).

[68] S. Obata, H. Tanaka, and K. Saiki, Carbon 55, 126 (2013).

[69] S. Obata and K. Saiki, Jpn. J. Appl. Phys. 58, 015003 (2019).

[70] H.-H. Huang, K. K. H. De Silva, G. R. A. Kumara, and M. Yoshimura, Sci. Rep. 8, 6849 (2018).

[71] B. Konkena and S. Vasudevan, J. Phys. Chem. Lett. 3, 867 (2012).

[72] X. Li, W. Cai, J. An, S. Kim, J. Nah, D. Yang, R. Piner, A. Velamakanni, I. Jung, E. Tutuc, S. K. Banerjee, L. Colombo, and R. S. Ruoff, Science 324, 1312 (2009).

[73] X. Li, W. Cai, L. Colombo, and R. S. Ruoff, Nano Lett. 9, 4268 (2009).

[74] L. Gao, W. Ren, H. Xu, L. Jin, Z. Wang, T. Ma, L.-P. Ma, Z. Zhang, Q. Fu, L.-M. Peng, X. Bao, and H.-M. Cheng, Nat. Commun. 3, 699 (2012).

[75] I. Pletikosić, M. Kralj, P. Pervan, R. Brako, J. Coraux, A. T. N'Diaye, C. Busse, and T. Michely, Phys. Rev. Lett. 102, 056808 (2009).

[76] P. W. Sutter, J.-I. Flege, and E. A. Sutter, Nat. Mater. 7, 406 (2008).

[77] J. K. Wassei, M. Mecklenburg, J. A. Torres, J. D. Fowler, B. C. Regan, R. B. Kaner, and B. H. Weiller, Small 8, 1415 (2012).

[78] X. Chen, P. Zhao, R. Xiang, S. Kim, J. Cha, S. Chiashi, and S. Maruyama, Carbon 94, 810 (2015).
[79] H. Kim, C. Mattevi, M. Reyes Calvo, J. C. Oberg, L. Artiglia, S. Agnoli, C. F. Hirjibehedin, M. Chhowalla, and E. Saiz, ACS Nano 6, 3614 (2012).

[80] I. Vlassiouk, M. Regmi, P. Fulvio, S. Dai, P. Datskos, G. Eres, and S. Smirnov, ACS Nano 5, 6069 (2011).

[81] X. Li, C. W. Magnuson, A. Venugopal, J. An, J. W. Suk, B. Han, M. Borysiak, W. Cai, A. Velamakanni, Y. Zhu, L. Fu, E. M. Vogel, L. Colombo, and R. S. Ruoff, Nano Lett. 10, 4328 (2010).

[82] P. Y. Huang, C. S. Ruiz-Vargas, A. M. van der Zande, W. S. Whitney, M. P. Levendorf, J. W. Kevek, S. Garg, J. S. Alden, C. J. Hustedt, Y. Zhu, J. Park, P. L. McEuen, and D. A. Muller, Nature 469, 389 (2011).

[83] Q. Yu, L. A. Jauregui, W. Wu, R. Colby, J. Tian, Z. Su, H. Cao, Z. Liu, D. Pandey, D. Wei, T. F. Chung, P. Peng, N. P. Guisinger, E. A. Stach, J. Bao, S.-S. Pei, and Y. P. Chen, Nat. Mater. 10, 443 (2011)

[84] X. Li, C. W. Magnuson, A. Venugopal, R. M. Tromp, J. B. Hannon, E. M. Vogel, L. Colombo, and R. S. Ruoff, J. Am. Chem. Soc. 133, 2816 (2011).

[85] Z. Yan, J. Lin, Z. Peng, Z. Sun, Y. Zhu, L. Li, C. Xiang, E. L. Samuel, C. Kittrell, and J. M. Tour, ACS Nano 6, 9110 (2012).

[86] L. Gan and Z. Luo, ACS Nano 7, 9480 (2013).

[87] S. Suzuki, K. Kiyosumi, T. Nagamori, K. Tanaka, and M. Yoshimura, e-J. Surf. Sci. Nanotechnol. 13, 404 (2015).

[88] S. Suzuki, Y. Terada, and M. Yoshimura, Coatings 7, 206 (2017).

[89] Y. Hao, M. S. Bharathi, L. Wang, Y. Liu, H. Chen, S. Nie, X. Wang, H. Chou, C. Tan, B. Fallahazad, H. Ramanarayan, C. W. Magnuson, E. Tutuc, B. I. Yakobson, K. F. McCarty, Y.-W. Zhang, P. Kim, J. Hone, L. Colombo, and R. S. Ruoff, Science 342, 720 (2013).

[90] L. Lin, J. Li, H. Ren, A. L. Koh, N. Kang, H. Peng, H. Q. Xu, and Z. Liu, ACS Nano 10, 2922 (2016).

[91] A. Reina, X. Jia, J. Ho, D. Nezich, H. Son, V. Bulovic, M. S. Dresselhaus, and J. Kong, Nano Lett. 9, 30 (2009).

[92] G. Deokar, J. Avila, I. Razado-Colambo, J.-L. Codron, C. Boyaval, E. Galopin, M.-C. Asensio, and D. Vignaud, Carbon 89, 82 (2015).

[93] H. J. Jeong, H. Y. Kim, S. Y. Jeong, J. T. Han, K.-J. Baeg, J. Y. Hwang, and G.-W. Lee, Carbon 66, 612 (2014).

[94] M. J. Allen, V. C. Tung, L. Gomez, Z. Xu, L.-M. Chen, K. S. Nelson, C. Zhou, R. B. Kaner, and Y. Yang, Adv. Mater. 21, 2098 (2009).

[95] J. D. Wood, G. P. Doidge, E. A. Carrion, J. C. Koepke, J. A. Kaitz, I. Datye, A. Behnam, J. Hewaparakrama, B. Aruin, and Y. Chen, Nanotechnology 26, 055302 (2015).

[96] Y. Wang, Y. Zheng, X. Xu, E. Dubuisson, Q. Bao, J. Lu, and K. P. Loh, ACS Nano 5, 9927 (2011).

[97] W.-H. Lin, T.-H. Chen, J.-K. Chang, J.-I. Taur, Y.-Y. Lo, W.-L. Lee, C.-S. Chang, W.-B. Su, and C.-I. Wu, ACS Nano 8, 1784 (2014).

[98] Y. Ren, C. Zhu, W. Cai, H. Li, Y. Hao, Y. Wu, S. Chen, Q. Wu, R. D. Piner, and R. S. Ruoff, Nano 7, 1150001 (2012).

[99] G. Zhang, A. G. Güell, P. M. Kirkman, R. A. Lazenby, T. S. Miller, and P. R. Unwin, ACS Appl. Mater. Interfaces 8, 8008 (2016)

[100] Y. Wu, P. Qiao, T. Chong, and Z. Shen, Adv. Mater. 14, 64 (2002).

[101] Y. Wang, H. Chen, M. Sun, Z. Yao, B. Quan, Z. Liu, Y. Weng, J. Zhao, C. Gu, and J. Li, Carbon 122, 98 (2017).

[102] A. K. Sivadasan, S. Parida, S. Ghosh, R. Pandian, and S. Dhara, Nanotechnology 28, 465703 (2017). 
[103] M. Zhu, J. Wang, B. C. Holloway, R. A. Outlaw, X. Zhao, K. Hou, V. Shutthanandan, and D. M. Manos, Carbon 45, 2229 (2007).

[104] K. Kobayashi, M. Tanimura, H. Nakai, A. Yoshimura, H. Yoshimura, K. Kojima, and M. Tachibana, J. Appl. Phys. 101, 094306 (2007).

[105] K. Tanaka, M. Yoshimura, A. Okamoto, and K. Ueda, Jpn. J. Appl. Phys. 44, 2074 (2005).

[106] S. Suzuki, A. Chatterjee, C.-L. Cheng, and M. Yoshimura, Jpn. J. Appl. Phys. 50, 01AF08 (2011).

[107] N. M. Santhosh, G. Filipič, E. Tatarova, O. Baranov, H. Kondo, M. Sekine, M. Hori, K. K. Ostrikov, and U. Cvelbar, Micromachines (Basel) 9, 565 (2018).

[108] J. Wang, M. Zhu, R. A. Outlaw, X. Zhao, D. M. Manos, and B. C. Holloway, Carbon 42, 2867 (2004).

[109] Y. Wu, B. Yang, B. Zong, H. Sun, Z. Shen, and Y. Feng, J. Mater. Chem. 14, 469 (2004).

[110] T. Itoh, S. Shimabukuro, S. Kawamura, and S. Nonomura, Thin Solid Films 501, 314 (2006).

[111] S. Shimabukuro, Y. Hatakeyama, M. Takeuchi, T. Itoh, and S. Nonomura, Jpn. J. Appl. Phys. 47, 8635 (2008).

[112] S. Shimabukuro, Y. Hatakeyama, M. Takeuchi, T. Itoh, and S. Nonomura, Thin Solid Films 516, 710 (2008).

[113] C. Yang, H. Bi, D. Wan, F. Huang, X. Xie, and M. Jiang, J. Mater. Chem. A 1, 770 (2013).

[114] G. Rius and M. Yoshimura, e-J. Surf. Sci. Nanotechnol. 10, 305 (2012).

[115] S. K. Srivastava, A. K. Shukla, V. D. Vankar, and V. Kumar, Thin Solid Films 492, 124 (2005).

[116] T. Machino, W. Takeuchi, H. Kano, M. Hiramatsu, and M. Hori, Appl. Phys. Express 2, 025001 (2009).

[117] M. Hiramatsu and M. Hori, Jpn. J. Appl. Phys. 45, 5522 (2006).

[118] K. Yu, Z. Bo, G. Lu, S. Mao, S. Cui, Y. Zhu, X. Chen, R. S. Ruoff, and J. Chen, Nanoscale Res. Lett. 6, 202 (2011).

[119] J. M. Gao, X. F. Song, J. Hu, S. C. Guo, L. Fang, F. Wu, and D. P. Wei, Mater. Lett. 184, 273 (2016).

[120] O. Tanaike, N. Kitada, H. Yoshimura, H. Hatori, K. Kojima, and M. Tachibana, Solid State Ionics 180, 381 (2009).

[121] C. S. Rout, A. Kumar, G. Xiong, J. Irudayaraj, and T. S. Fisher, Appl. Phys. Lett. 97, 133108 (2010).

[122] C. S. Rout, A. Kumar, and T. S. Fisher, Nanotechnology 22, 395704 (2011).

[123] P. Dyakonov, K. Mironovich, S. Svyakhovskiy, O. Voloshina, S. Dagesyan, A. Panchishin, N. Suetin, V. Bagratashvili, P. Timashev, and E. Shirshin, Sci. Rep. 7, 13352 (2017).

[124] J. Zhao, M. Sun, Z. Liu, B. Quan, C. Gu, and J. Li, Sci. Rep. 5, 16019 (2015).
[125] X. Xiu, Y. Guo, C. Li, Z. Li, D. Li, C. Zang, S. Jiang, A. Liu, B. Man, and C. Zhang, Opt. Mater. Express 8, 844 (2018).

[126] C.-Y. Zhang, R. Hao, B. Zhao, Y.-W. Hao, and Y.-Q. Liu, Appl. Surf. Sci. 409, 306 (2017).

[127] J. Huang, L. Zhang, B. Chen, N. Ji, F. Chen, Y. Zhang, and Z. Zhang, Nanoscale 2, 2733 (2010).

[128] Y. Jiang, J. Wang, L. Malfatti, D. Carboni, N. Senes, and P. Innocenzi, Appl. Surf. Sci. 450, 451 (2018).

[129] Z. Zhang, F. Xu, W. Yang, M. Guo, X. Wang, B. Zhang, and J. Tang, Chem. Commun. 47, 6440 (2011).

[130] Y. Zhang, S. Liu, L. Wang, X. Qin, J. Tian, W. Lu, G. Chang, and X. Sun, RSC Adv. 2, 538 (2012).

[131] K. Jasuja and V. Berry, ACS Nano 3, 2358 (2009).

[132] S. Xu, L. Yong, and P. Wu, ACS Appl. Mater. Interfaces 5, 654 (2013).

[133] W. Ren, Y. Fang, and E. Wang, ACS Nano 5, 6425 (2011).

[134] Y.-K. Kim, G. Ok, S.-W. Choi, H. Jang, and D.-H. Min, Nanoscale 9, 5872 (2017).

[135] S. Suzuki and M. Yoshimura, Sci. Rep. 7, 14851 (2017).

[136] X. Zhu, L. Shi, M. S. Schmidt, A. Boisen, O. Hansen, J. Zi, S. Xiao, and N. A. Mortensen, Nano Lett. 13, 4690 (2013).

[137] P. Wang, O. Liang, W. Zhang, T. Schroeder, and Y.-H. Xie, Adv. Mater. 25, 4918 (2013).

[138] X. Li, W. C. H. Choy, X. Ren, D. Zhang, and H. Lu, Adv. Funct. Mater. 24, 3114 (2014).

[139] J. Leem, M. C. Wang, P. Kang, and S. Nam, Nano Lett. 15, 7684 (2015)

[140] S. Xu, S. Jiang, J. Wang, J. Wei, W. Yue, and Y. Ma, Sens. Actuators B Chem. 222, 1175 (2016).

[141] H. Qiu, M. Wang, S. Jiang, L. Zhang, Z. Yang, L. Li, J. Li, M. Cao, and J. Huang, Sens. Actuators B Chem. 249, 439 (2017).

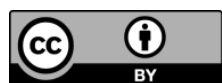

All articles published on e-J. Surf. Sci. Nanotechnol. are licensed under the Creative Commons Attribution 4.0 International (CC BY 4.0). You are free to copy and redistribute articles in any medium or format and also free to remix, transform, and build upon articles for any purpose (including a commercial use) as long as you give appropriate credit to the original source and provide a link to the Creative Commons (CC) license. If you modify the material, you must indicate changes in a proper way.

Published by The Japan Society of Vacuum and Surface Science 